\title{
Is it cardiac involvement mimicking acute myocardial infarction in idiopathic inflammatory myopathy?
}

\author{
YIZHI XIAO, HUI LUO, SHIQING LIU, LIPING DUAN, YUNHUI YOU, \\ HONGJUN ZHAO and XIAOXIA ZUO \\ Department of Rheumatology and Immunology, Xiangya Hospital, \\ Central South University, Changsha, Hunan 410008, P.R. China \\ Received February 18, 2016; Accepted February 7, 2017
}

DOI: $10.3892 / \mathrm{etm} .2017 .4447$

\begin{abstract}
Idiopathic inflammatory myopathy (IIM) comprises a group of rare systemic diseases characterized by progressive weakness of the symmetrical proximal limb muscles, elevated muscle enzymes, inflammation or necrosis on muscle biopsy. IIM may impair the function of multiple organs, particularly the heart. However, it rarely manifests as acute myocardial infarction (AMI) at initial presentation. The present study described the case of a 39-year-old woman with AMI, whose muscle biopsy on the left arm conformed to polymyositis. Coronary arteriography showed irregularities in the left descending and right coronary artery (25\% diameter reduction in middle segment). It was concluded that AMI was the chief manifestation of IIM at initial presentation. Furthermore, to the best of our knowledge, the present study was the first to provide a systematic literature review to assess AMI in IIM patients and discuss the possible causes of AMI.
\end{abstract}

\section{Introduction}

Idiopathic inflammatory myopathy (IIM) defines a group of non-suppurative inflammatory diseases comprising an immune-mediated attack on skeletal muscle, leading to muscle damage and weakness in the patient. The adjusted annual incidence of IIM in the USA ranges from 5.8-7.9 per 100,000 individuals (1).

Dermatomyositis (DM), polymyositis (PM) and inclusion body myositis (IBM) are the most common IIM subtypes in clinical practice. However, the mechanisms of IIM have remained to be fully elucidated. IIM is accompanied by impaired function of multiple organs, particularly the heart, resulting in a poor prognosis. Cardiac manifestations

Correspondence to: Dr Hongjun Zhao, Department of Rheumatology and Immunology, Xiangya Hospital, Central South University, 87 Xiangya Road, Changsha, Hunan 410008, P.R. China E-mail: hongjunzhao2015@sina.com

Key words: idiopathic inflammatory myopathy, polymyositis, dermatomyositis, acute myocardial infarction constitute a certain percentage of the causes of myositis-associated death $(2,3)$. Cardiac manifestations of IIM have been observed on electrocardiography (ECG) in up to $72 \%$ of cases and include tachycardia, arrhythmias, blocks, abnormal Q-waves and non-specific ST-T wave changes. Pericardial effusion, atrial and ventricular enlargement as well as hypokinesis are found on echocardiography. However, most of the cardiac involvement is nonspecific and subclinical (4-8). Cardiac involvement is encountered with a high incidence in IIM patients, but rarely manifests as acute myocardial infarction (AMI) at initial presentation. The present study described a case with AMI at initial presentation of PM. A systematic literature review on AMI in IIM patients was also included.

\section{Materials and methods}

Case presentation. A 39-year-old woman was referred to the Department of Rheumatology and Immunology of Xiangya Hospital (Changsha, China) on March 20, 2013 with a history of edema, feebleness, post-exercise tachypnea persisting for half a month and dysphagia for 4 days. She complained of sudden mild precordial discomfort without chest tightness or pain lasting for several hours during the first night of hospitalization. She did not have any history of chest trauma or long-term use of any medication.

Initial physical examination revealed that her pulse, blood pressure and respiration were 100/min, 98/64 $\mathrm{mmHg}$ and 20/min, respectively. Neck vein distention and examination of the lung and heart yielded normal findings. The lower limbs contained pitting edema, the muscle strength of the upper extremities was grade 4 and that of the lower extremities was grade 3 , most commonly proximal.

Laboratory examination showed anemia [hemoglobin, $86 \mathrm{~g} / \mathrm{l}$ (normal reference value, 110-150 g/l)] and low complement level supported by a decrease of C4 [105 mg/l (normal reference value, $120-360 \mathrm{mg} / \mathrm{l}$ )] and C3 [580 mg/l (normal reference value, 850-1390 mg/l)]. Cardiac troponin I (cTnI) levels (normal reference value, $<0.16 \mathrm{pg} / \mathrm{ml}$ ) and myocardial enzymology values (normal reference value: Lactate dehydrogenase, LDH: 109-245 U/l; creatine kinase, CK: 24-190 U/1; CK MB isoenzyme, CK-MB: <24 U/1; myoglobin, Mb: $<70 \mu \mathrm{g} / \mathrm{l}$ ) were raised (Figs. 1 and 2). The level of N-terminal 


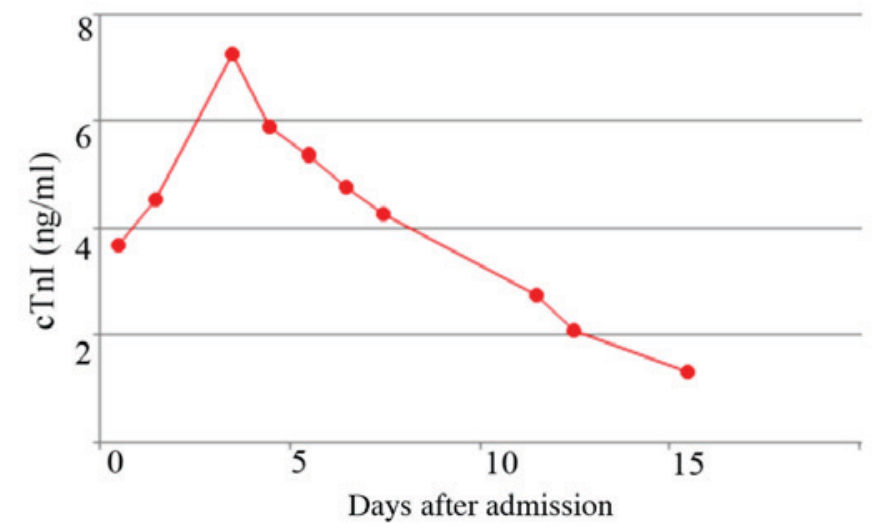

Figure 1. cTnI levels in the 39-year-old female patient with myocardial involvement mimicking acute myocardial infarction. cTnI, cardiac troponin I (normal reference value, $<0.16 \mathrm{pg} / \mathrm{ml}$ ).

pro-brain natriuretic peptide was $1,947 \mathrm{pg} / \mathrm{m}$; (normal reference value $<250 \mathrm{pg} / \mathrm{ml}$ ) indicating a poor cardiac function. The patient was negative for detectable autoantibodies, including antinuclear antibodies, anti-double-stranded DNA, anti-Smith, anti-Sjögren's syndrome (SS)A, anti-SSB, anti-Jo-1, anti-Scl-70 and anti-nuclear ribonucleoprotein. The white blood cell count, platelet count and erythrocyte sedimentation rate were all normal.

Muscle biopsy on the left arm conformed to PM. The 18-lead ECG on admission revealed sinus rhythm and ST-segment sloop down in leads V1-V3 (Fig. 3). The cardiac form, structure, functionality and valve activities were normal on cardiac Doppler ultrasonography. Coronary arteriography was performed on admission, March 20, 2013 and showed irregularities in the left anterior descending and right coronary artery (25\% diameter reduction in the middle segment). However, no culprit lesion was found (Fig. 4).

Based on of all the above data, a diagnosis of PM with cardiac involvement was made. The patient was given methylprednisolone (500 mg/day), aspirin ( $0.1 \mathrm{~g}$ per night), together with sodium nitroprusside and cedilanid to treat cardiac failure. During the treatment, the patient showed recurrent dyspnea and chest discomfort, and succumbed to respiratory and circulatory failure 20 days later, although the myocardial enzymology and cTnI levels had markedly decreased.

Literature review. In addition to the case reported in the present study, all available previous studies were retrieved through a systematic review on AMI in IIM. Studies published in indexed international journals included in the PubMed database from January 1970 to January 2017 were analyzed. Cases of IIM patients who were diagnosed with AMI and with sufficient information provided were included. Studies published in the English language were selected and additional cross-checks of references cited in them were performed. As the search strategy, a combination of the following terms was used: 'Idiopathic inflammatory myopathy', 'polymyositis' or 'dermatomyositis' and 'acute myocardial infarction', and 6 studies were retrieved. A total of 6 cases of AMI in IIM were reported (9-14) (Table I).

The cases comprised $3 \mathrm{PM}$ and $3 \mathrm{DM}$ patients, including 4 females and 2 males (each of which had DM), and no IBM was reported (9-14). In cases 2 and 4-6, the course of IIM was long and they were administered prednisolone at various doses, while case 3 had an acute onset. Case 1 received metoprolol (95 mg/day) and simvastatin (20 mg/day) for hypertension and hypercholesterolemia, respectively, prior to admission (13). Case 3 had a history of blunt chest trauma due to an automobile accident (10). Case 5 had received thrombolysis as a myocardial infarction therapy 2 weeks prior to admission (11).

Case 2, 5 and 6 had complaints of chest discomfort or chest pain $(9,11,14)$, while case 4 mainly presented with vomiting followed by syncope (12). Case 1 and 3 did not show any symptoms in the heart $(10,13)$. Only case 1 was subjected to cTnI examination and showed a mild increase (13). All cases were negative for antinuclear antibodies. Except for case 5, coronary angiography showed small changes in all cases, which may not have been associated with the severe chest symptoms (9-14). Cases 1 and 2 showed occlusive spasm after intracoronary acetylcholine provocation for vasospasm on

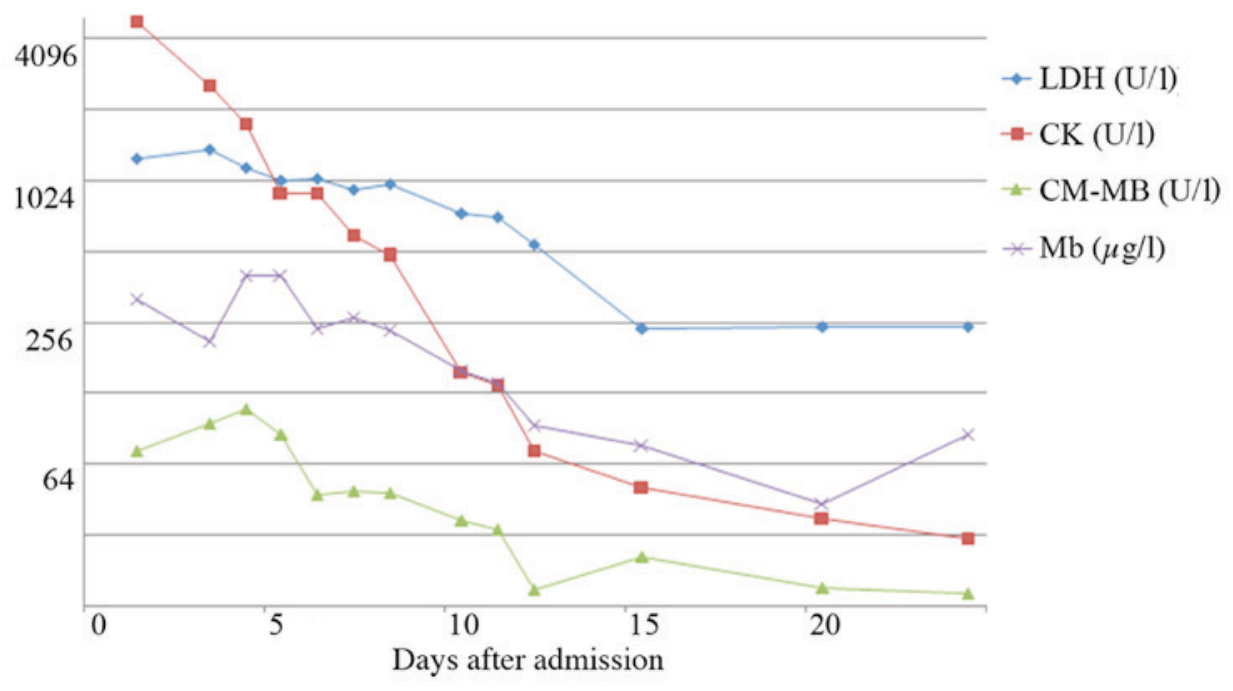

Figure 2. Myocardial enzymology results showed variations with time. Normal reference values: LDH, 109-245 U/1; CK, 24-190 U/1; CK-MB, <24 U/1; Mb, $<70 \mu \mathrm{g} / \mathrm{l}$. LDH, lactate dehydrogenase; CK, creatine kinase; CKMB, CK MB isoenzyme; Mb, myoglobin. 


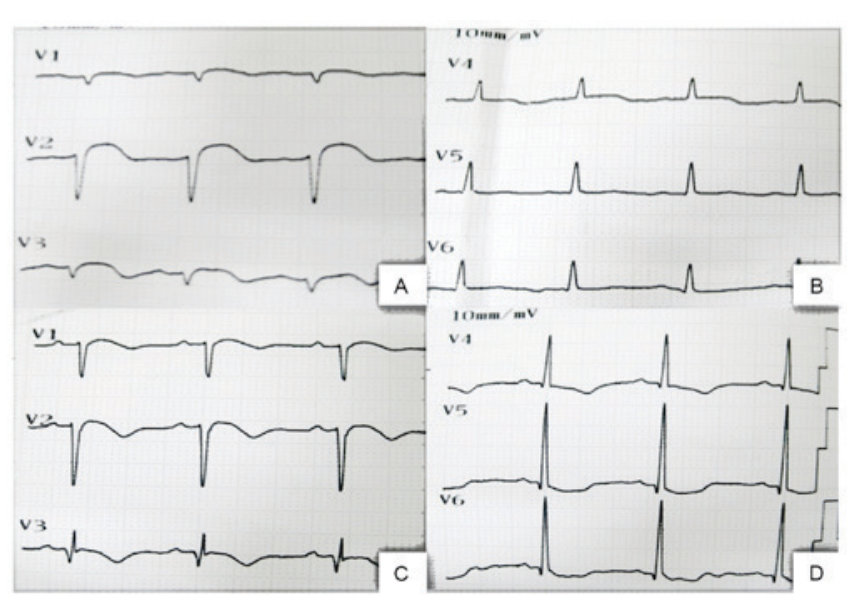

Figure 3.ECGs of the 39-year-old female patient with myocardial involvement mimicking acute myocardial infarction. (A and B) The ECG on admission (March 20,2013) revealed sinus rhythm and ST-segment sloop down in leads V1-V3. (C and D) The ECG on admission (March 27, 2013) revealed sinus rhythm and ST-segment elevation in leads V1-V2. ECG, electrocardiogram.

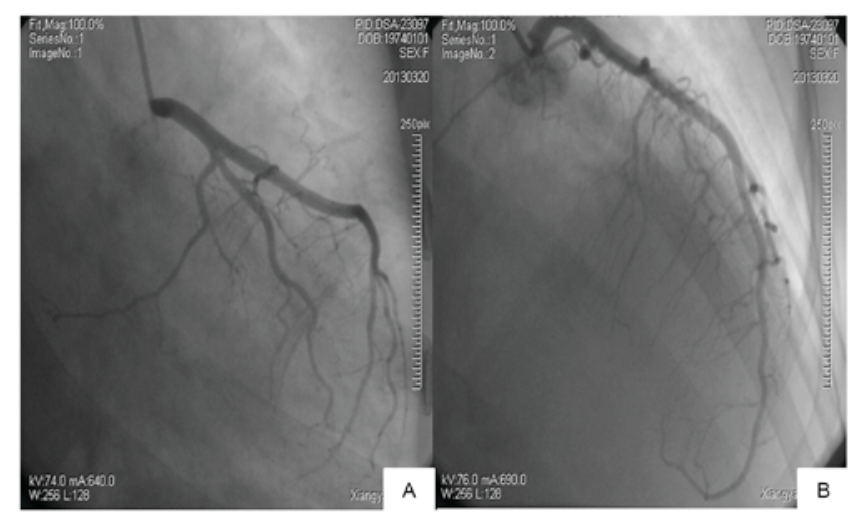

Figure 4. Coronary arteriographs of the 39-year-old female patient with myocardial involvement mimicking acute myocardial infarction. (March 20, 2013). A 25\% diameter reduction in the middle segment of the (A) left anterior descending coronary artery and (B) right coronary artery.

coronary angiography $(13,14)$. Coronary angiography of case 5 showed a left anterior descending coronary artery (LAD) dissection from the mid-to-distal part with $95 \%$ stenosis (11). The diagnosis of spontaneous coronary artery dissection causing AMI was made and the condition was successfully managed with percutaneous coronary intervention and stent placement.

Cases 2-6 responded well to treatment $(9-12,14)$. Case 2 suffered recurrent severe chest pain and only added calcium antagonism with amlodipine $5 \mathrm{mg}$ /day markedly improved anginal symptoms requiring no further hospital admissions for angina (14). Most studies considered the cause of the symptoms to be inflammatory processes due to IIM and vasoconstriction due to impaired regulation of abnormal vasomotion $(9,10,12-14)$.

\section{Discussion}

IIMs are a group of rare systemic diseases, which frequently show cardiac manifestations, which is, however, subclinical in most cases. In 1979, Cohn and Lynfield (10) first reported on an IIM patient who presented with AMI, who was included in the present literature analysis as case 3. Case 2, 5 and 6 had a long history of IIM before the onset of AMI and suffered an exacerbation $(9,11,14)$. In 2009, Tisseverasinghe et al $(15)$ reported a high incidence of arterial events in IIM patients. A longitudinal follow-up study by Lai et al (16) demonstrated that DM is associated with an increased risk of cardiovascular events, particularly AMI. Other studies confirmed this finding (17-19); however, the specific risk factors of AMI in IIM patients have remained to be investigated. Flow-mediated dilatation of the brachial artery, arterial stiffness and carotid artery thickness on ultrasonography may be predictors of cardiovascular disease in IIM patients (18). Case 4, who suffered a left hemispheric infarction 9 years after AMI, indicated that not only cardiovascular but also cerebrovascular disease poses a high risk $(12,16)$.

Furthermore, case 1, 2, 5 and 6 received prednisolone treatment prior to the onset of AMI. Administration of glucocorticoids at high doses or for a long time is a risk factor for diabetes mellitus, hypertension and hyperlipidemia, resulting in atherosclerosis or cardiovascular disease, particularly coronary heart disease (20). This may in part explain for the high infarction risk in IIM patients (16-19). Glucocorticoid dosages should be gradually reduced when the disease can be controlled.

As shown in Table I, IIM was in the active state in numerous patients with AMI, which possibly presented secondarily to the cardiac involvement of IIM $(10,12,13)$. In 1990, Emslie-Smith and Engel (21) reported that small arterioles, capillaries and venules are early and specific targets of the pathological process in DM. Inflammatory cells such as macrophages, activated-T lymphocytes and dendritic cells, located around blood vessels in the perimysium have been reported to cause endothelial dysfunction $(22,23)$. In addition, chronic inflammation contributes to coagulation by upregulating pro-coagulants, downregulating anticoagulants and suppressing fibrinolysis $(23,24)$. Vasculopathy and a high coagulation state may result in small artery stenosis and lead to infarction, which has been observed in numerous organs, including the brain, retina, kidney and spleen $(25,26)$. Chronic inflammation has been demonstrated to be associated with other autoimmune diseases. Furthermore, changes in progesterone may weaken vessels in peripartum females with IIM $(11,27)$. All of these factors affect the arterioles in the perimysium of cardiac muscle.

Acetylcholine testing by coronary angiography was performed in case 1 and $2(13,14)$. An interesting phenomenon was that while occlusive spasm is at times found after acetylcholine testing, coronary angiography showed only mild diameter reduction $(13,14)$. The abnormalities on angiography returned to normal after intracoronary nitroglycerine administration. Certain inflammatory pathways, as well as high expression of adhesion molecules due to elevated C-reactive protein concentrations, and expansion of CD $4{ }^{+} \mathrm{CD} 28^{\text {null }} \mathrm{T}$ cells, result in damage of endothelial cells and early atherosclerosis, and lead to abnormal vasomotion by increasing endothelin-1 and reducing NO production. This may provide an explanation for the occurrence of coronary artery spasm (13). Furthermore, inflammatory cells such as $\mathrm{T}$ cells, macrophages and mast cells are all important effector cells that participate in the 


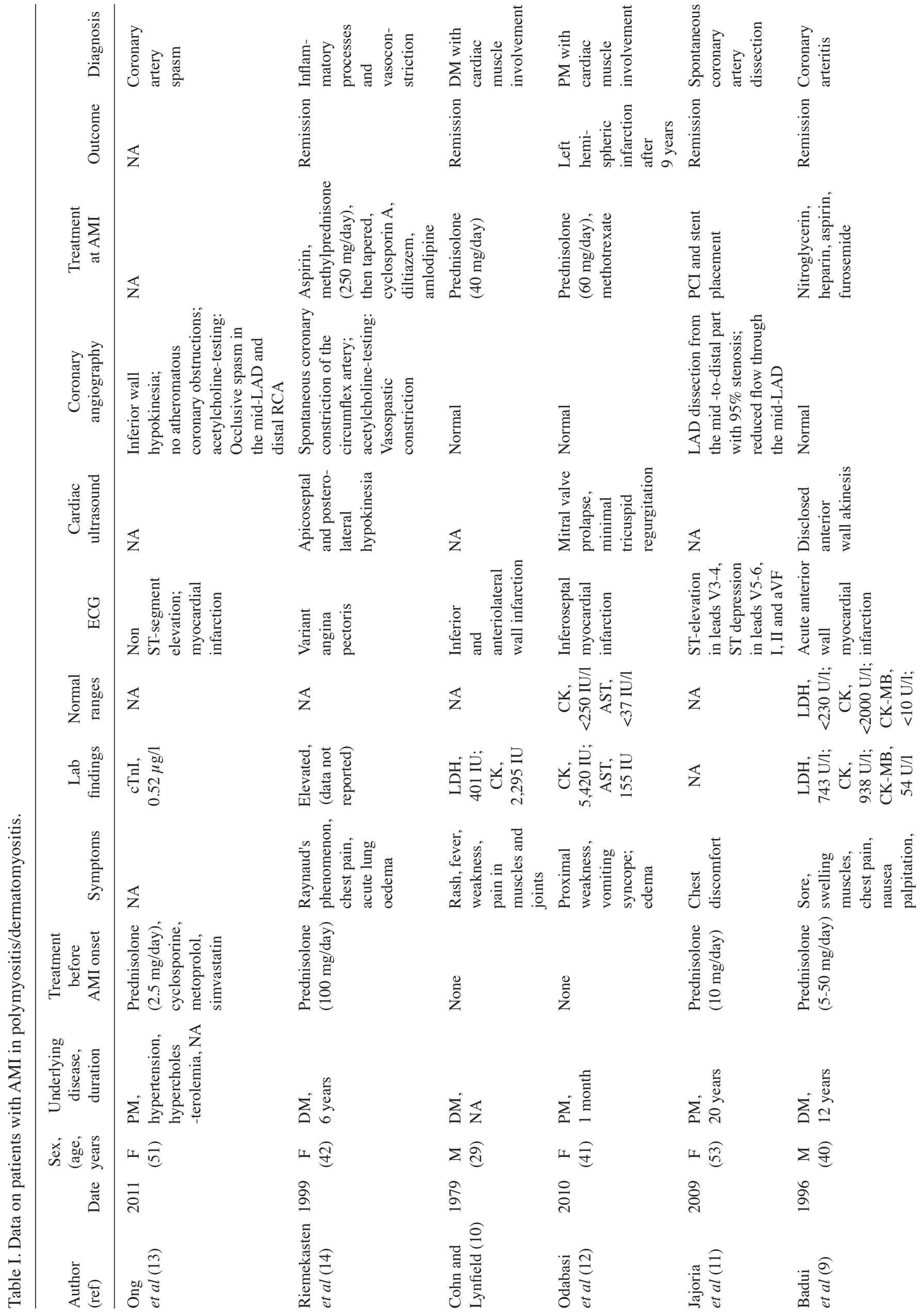


pathogenesis of inflammatory hypersensitivity disease and coronary artery spasm (28).

IIM has a feature of muscle enzyme elevation with regard to creatine kinase (CK), CK MB isoform, lactate dehydrogenase and myoglobin. It is difficult to distinguish between simple IIM and IIM with AMI when the clinical manifestations are untypical. cTn, composed of the three subunits cTnT, cTnI and cTnC, is a sensitive laboratory parameter associated with heart involvement. cTnT but not cTnI, along with CK, was reported to be significantly higher in IIM with heart involvement $(2,29-31)$. Only in a small percentage of IIM patients, mild elevation of cTnI is observed, although it is a sensitive laboratory parameter associated with cardiac muscle damage. cTnI drops rapidly along with myocardial enzymes and the ST-segment is depressed on ECG after administration of aggressive glucocorticoid and anti-heart-failure treatment, demonstrating that cTnI varies with the activity of AMI. The present case showed the marked elevation of $\mathrm{cTnI}$ on admission and a continuous high level of cTnI, which cannot explained by specific drugs, therefore considered to be indicative of AMI.

The case of the present study was a middle-aged woman with complaints of persistent chest discomfort, who had no risk factors for CAD and had no history of corticosteroid intake for prolonged periods. She was unique in that not only her ECG but also the dynamic changes of cTnI revealed an anterior wall myocardial infarction, while coronary angiography revealed no severe stenosis. Except for case 5, where spontaneous coronary artery dissection led to stenosis, case 1-4 and 6 showed a similar phenomenon: Coronary angiography did not conform to the findings of clinical manifestation, and $\mathrm{cTnI}$ and ECG revealed AMI. It remains elusive whether the case of the present study suffered real AMI or any other cardiac event mimicking AMI of unknown cause. Furthermore, it is unknown why AMI only affected the anterior wall of the patient's heart. Coronary artery spasm may be one of the causes of the manifestation of AMI $(13,14)$. However, based on the following facts, vasospasm does not fully explain the changes in cTnI, myocardial enzymes and ECG findings, particularly in the case of the present study: Coronary angiography revealed no severe stenosis, and muscle enzyme and troponin showed a marked decline after pulse therapy with methylprednisolone. Therefore, the present case is more likely to have presented with myocardial involvement mimicking AMI during an exacerbation of IIM.

To the best of our knowledge, the present study provided the first literature on myocardial involvement mimicking AMI in IIM. However, it has limitations mainly due to the rarity of the condition and the number of patients reviewed. While a few cases of AMI were reported between 1970 and 1998, they lack certain sensitive laboratory examinations of heart involvement such as Tn (23). Furthermore, most of the case reports retrieved described patients with a favorable response, while those with poor outcomes were rarely reported. In addition, no autopsy was performed on the patient of the present study to confirm the conjecture made.

According to various studies, IIM patients are at risk of coronary heart disease and myocardial involvement mimicking AMI may be a rare phenomenon in IIM patients. Myocardial involvement mimicking AMI may be the chief manifestation of IIM at initial presentation in the active stage; however, the 
exact mechanism remains elusive and requires further study. cTnI may be a sensitive laboratory marker and the acetylcholine test in coronary angiography is recommended. It is necessary to highlight the importance of vigilance regarding cardiovascular diseases in the management of patients with IIM. Therefore, medical staff is required to pay more attention to cardiovascular diseases in clinical practice.

\section{References}

1. Furst DE, Amato AA, Iorga ȘR, Gajria K and Fernandes AW: Epidemiology of adult idiopathic inflammatory myopathies in a U.S. Managed care plan. Muscle Nerve 45: 676-683, 2012.

2. Danko K, Ponyi A, Constantin T, Borgulya G and Szegedi G: Long-term survival of patients with idiopathic inflammatory myopathies according to clinical features: A longitudinal study of 162 cases. Medicine 83: 35-42, 2004.

3. Sultan SM, Ioannou Y, Moss K and Isenberg DA: Outcome in patients with idiopathic inflammatory myositis: Morbidity and mortality. Rheumatology (Oxford) 41: 22-26, 2002.

4. Agrawal CS, Behari M, Shrivastava S, Ahuja GK, Bhandari S and Kothari SS: The heart in polymyositis-dermatomyositis. J Neurol 236: 249-250, 1989.

5. Gottdiener JS, Sherber HS, Hawley RJ and Engel WK: Cardiac manifestations in polymyositis. Am J Cardiol 41: 1141-1149, 1978

6. Lundberg IE: The heart in dermatomyositis and polymyositis. Rheumatology (Oxford) 45 (Suppl 4): iv18-iv21, 2006.

7. Taylor AJ, Wortham DC, Burge JR and Rogan KM: The heart in polymyositis: A prospective evaluation of 26 patients. Clin Cardiol 16: 802-808, 1993.

8. Yazici Y and Kagen LJ: Cardiac involvement in myositis. Curr Opin Rheumatol 14: 663-665, 2002.

9. Badui E, Valdespino A, Lepe L, Rangel A, Campos A and Leon F: Acute myocardial infarction with normal coronary arteries in a patient with dermatomyositis. Case report. Angiology 47: 815-818, 1996

10. Cohn H and Lynfield YL: Myocardial infarction in dermatomyositis. Cutis 23: 672-675, 1979.

11. Jajoria P, Tuero EI and Lui CY: Spontaneous coronary artery dissection causing acute myocardial infarction in a post-menopausal woman with rheumatological disorder (polymyositis): Treatment dilemma. J Invasive Cardiol 21: E132-E133, 2009.

12. Odabasi Z, Yapundich R and Oh SJ: Polymyositis presenting with cardiac manifestations: Report of two cases and review of the literature. Clin Neurol Neurosurg 112: 160-163, 2010.

13. Ong P, Athanasiadis A, Alscher MD, Fritz P, Mahrholdt H, Sechtem U and Kaski JC: Coronary artery spasm as a cause for myocardial infarction in patients with systemic inflammatory disease. Int J Cardiol 151: e32-e34, 2011.

14. Riemekasten G, Opitz C, Audring H, Barthelmes H, Meyer R, Hiepe F and Burmester GR: Beware of the heart: The multiple picture of cardiac involvement in myositis. Rheumatology (Oxford) 38: 1153-1157, 1999.

15. Tisseverasinghe A, Bernatsky $\mathrm{S}$ and Pineau CA: Arterial events in persons with dermatomyositis and polymyositis. J Rheumatol 36 : 1943-1946, 2009.
16. Lai YT, Dai YS, Yen MF, Chen LS, Chen HH, Cooper RG and Pan SL: Dermatomyositis is associated with an increased risk of cardiovascular and cerebrovascular events: A Taiwanese population-based longitudinal follow-up study. Br J Dermatol 168: 1054-1059, 2013.

17. Linos E, Fiorentino D, Lingala B, Krishnan E and Chung L: Atherosclerotic cardiovascular disease and dermatomyositis: An analysis of the nationwide inpatient sample survey. Arthritis Res Ther 15: R7, 2013.

18. Vincze M, Dér H, Kerekes G, Szodoray P, Zeher M, Dankó K and Soltész P: Decreased flow-mediated dilatation with increased arterial stiffness and thickness as early signs of atherosclerosis in polymyositis and dermatomyositis patients. Clin Rheumatol 33: 1635-1641, 2014.

19. Ungprasert P, Suksaranjit P, Spanuchart I, Leeaphorn N and Permpalung N: Risk of coronary artery disease in patients with idiopathic inflammatory myopathies: A systematic review and meta-analysis of observational studies. Semin Arthritis Rheum 44: 63-67, 2014.

20. Sholter DE and Armstrong PW: Adverse effects of corticosteroids on the cardiovascular system. Can J Cardiol 16: 505-511, 2000.

21. Emslie-Smith AM and Engel AG: Microvascular changes in early and advanced dermatomyositis: A quantitative study. Ann Neurol 27: 343-356, 1990.

22. Amato AA and Greenberg SA: Inflammatory myopathies. Continuum (Minneap Minn) 19: 1615-1633, 2013.

23. Esmon CT: The interactions between inflammation and coagulation. Br J Haematol 131: 417-430, 2005.

24. Xu J, Lupu F and Esmon CT: Inflammation, innate immunity and blood coagulation. Hamostaseologie 30: 5-6, 8-9, 2010.

25. De Vries S: Retinopathy in dermatomyositis. AMA Arch Ophthalmol 46: 432-435, 1951

26. Matsuda Y, Harigai M, Nakajima $H$, Terajima $H$, Yamada $T$, Fukasawa C, Takeuchi $M$, Hara $M$ and Kamatani $N$ : Dermatomyositis with splenic and renal infarctions during corticosteroid therapy. Intern Med 39: 512-516, 2000.

27. Barrett JM, Van Hooydonk JE and Boehm FH: Pregnancy-related rupture of arterial aneurysms. Obstet Gynecol Surv 37: 557-566, 1982.

28. Almpanis GC, Kounis GN, Mazarakis A and Kounis NG: Coronary artery spasm progressing to acute myocardial infarction in patients with systemic inflammatory disease: A potential association with kounis syndrome. Int J Cardiol 151: 1-2, 2011.

29. Aggarwal R, Lebiedz-Odrobina D, Sinha A, Manadan A and Case JP: Serum cardiac troponin T, but not troponin I, is elevated in idiopathic inflammatory myopathies. J Rheumatol 36: 2711-2714, 2009.

30. Fisher C, Agrawal S, Wong WM, Fahie-Wilson M and Dasgupta B: Clinical observations on the significance of raised cardiac troponin-T in patients with myositis of varying etiologies seen in rheumatology practice. Clin Rheumatol 29: 1107-1111, 2010.

31. Gerhardt W and Ljungdahl L: Troponin T: A sensitive and specific diagnostic and prognostic marker of myocardial damage. Clin Chim Acta 272: 47-57, 1998. 